\title{
Dificuldades para a implantação sistematização da assistência de enfermagem em maternidades
}

Objetivou-se descrever as dificuldades enfrentadas por docentes e discentes de enfermagem da Universidade Federal do Piauí para a implantação da SAE em uma maternidade. Nesta reflexão encontraram-se como dificuldades: não preenchimento dos impressos, descontinuidade da assistência, falta de comunicação entre equipe e acadêmicos, deficiência da educação permanente, falta de preparo dos profissionais e número insuficiente de pessoal. Há necessidade de uma maior coesão entre a equipe de enfermagem, em busca de benefícios para a profissão, cliente e instituição. Espera-se, com este estudo, trazer contribuições aos profissionais de enfermagem no sentido de incentivar a realização de uma assistência digna, humanizada e sistematizada.

Descritores: Assistência de Enfermagem, Saúde da Mulher, Docentes.

\section{Difficulties in the implementation of nursing care systematization (SAE) in maternities}

This work aimed to describe the difficulties faced by the faculty and students of nursing of the Federal University of Piauí in the implementation of the SAE in a maternity. The difficulties in this regard were: non-filling of the papers, discontinuance of the care service, lack of communication between the team and university students, deficiency in the permanent education, lack of preparation of the professionals and insufficient number of personnel. There is a need for higher cohesion between the nursing team, towards benefits for the profession, client, and institution. This study is expected to bring some inputs for the nursing professionals in order to promote a worthy, humanized, and systematized care service.

Descriptors: Nursing Care, Women's Health, Faculty.

\section{Dificultades para la implantación y sistematización de la asistencia de enfermería en maternidades}

El objetivo fue el de describir dificultades enfrentadas por docentes y discentes de enfermería de la Universidad Federal de Piauí para la implantación de la SAE en una maternidad. En esta reflexión se encuentran como dificultades: el no llenado de los impresos, discontinuidad de la asistencia, falta de comunicación entre el equipo y los académicos, deficiencia de la educación permanente, falta de preparación de los profesionales y número insuficiente de personal. Existe la necesidad de una mayor cohesión entre el equipo de enfermería, en busca de beneficios para la profesión, cliente e institución. Se espera, con este estudio, traer contribuciones a los profesionales de enfermería en el sentido de incentivar la realización de una asistencia digna, humanizada y sistematizada.

Descriptores: Asistencia de Enfermería, Salud de la Mujer, Docentes.

\section{INTRODUÇÃO}

A Sistematização da Assistência de Enfermagem (SAE) é a utilização de método e estratégia de trabalho científico para a identificação das situações de saúde/ doença, que embasam as ações de assistência de Enfermagem e que contribuem para a promoção, prevenção, recuperação e reabilitação da saúde do indivíduo, família e comunidade. Em se tratando de cuidados para mulheres no ciclo grávido-puerperal, a SAE assume especial importância, pois alterações psíquicas e fisiológicas próprias da gestação distinguem essas mulheres dos demais indivíduos que não se encontram nessa fase. Além disso, complicações próprias da gravidez merecem uma assistência individualizada por parte da equipe de saúde ${ }^{(1-2)}$.

É coerente, desta forma, afirmar que quanto maior o

1 Enfermeira Obstétrica. Doutora em Enfermagem. Professora Associada II do Curso de Enfermagem e membro efetivo do Programa de Mestrado em Enfermagem e do Mestrado/ Doutorado de Políticas Públicas da Universidade Federal do Piauí. Teresina- PI. Email: ineznery.ufpi@gmail.com

2 Enfermeira. Especialista em Urgência e Emergência. Mestranda em Ciências e Saúde na Universidade Federal do Piauí. Teresina- PI.

3 Enfermeira. Mestre em Enfermagem. Professora do Curso de Enfermagem da Universidade Federal do Piauí (aposentada). Conselheira Presidente do Coren-PI Gestões 19992002 e 2005-2011. Coordenadora da Comissão de Publicação da ABEn-Secção Piauí; Conselheira Efetiva do COFEN, e Membro da Câmara Técnica de Sistematização da Assistência de Enfermagem do COFEN. 
número de necessidades humanas básicas afetadas durante a gravidez, parto e puerpério, maior é a necessidade de se planejar a assistência, uma vez que a sistematização das ações visa a organização, eficiência e validade da assistência prestada. Assim, incorporar a SAE à assistência é uma forma de tornar a Enfermagem mais científica, promovendo um cuidar de enfermagem humanizado, contínuo, mais justo e com qualidade para a paciente ${ }^{(3)}$.

No que se refere aos aspectos legais, em 2002, por meio da Resolução COFEN no 272/2002, tornou-se obrigatória a implantação e implementação da Sistematização da Assistência de Enfermagem em todas as instituições de saúde públicas e privadas, determinando-se então as fases para a sua operacionalização. Em 2009, essa resolução foi revogada pela Resolução COFEN no 358/2009, a qual determina que o Processo de Enfermagem deve ser realizado de modo deliberado e sistemático em todos os ambientes em que ocorre o cuidado profissional de Enfermagem, incluindo hospitais, serviços ambulatoriais, escolas e domicílio; e que deve se organizar em cinco etapas interrelacionadas, interdependentes e recorrentes, a saber: histórico de enfermagem, diagnóstico de enfermagem, planejamento de enfermagem, implementação e avaliação de enfermagem ${ }^{(4-5)}$.

No entanto, apesar de ser obrigatória, a sua aplicação, as etapas do processo de enfermagem não são realizadas de forma adequada pelo enfermeiro; muitas vezes, devido à sobrecarga de trabalho imposta a esse profissional, que acaba se detendo em atividades burocráticas e administrativas, que também fazem parte de suas atribuições profissionais ${ }^{(6)}$.

Esta é uma realidade que requer reflexões, pois é a partir da sistematização da assistência que o paciente poderá ser visto de maneira holística, mais humana e individual. Dessa forma pode-se afirmar que a SAE é uma estratégia por meio da qual a Enfermagem desenvolverá um trabalho humanizado, qualificado e individualizado.

Assim, este estudo teve como objetivos: descrever aspectos referentes às dificuldades enfrentadas por docentes e discentes de enfermagem da Universidade Federal do Piauí (UFPI) para a implantação da SAE em uma maternidade pública de referência no atendimento à mulher no ciclo gravídico-puerperal.

\section{METODOLOGIA}

Este estudo consiste em uma reflexão acerca da experiência vivenciada na implantação da sistematização da assistência de enfermagem por docentes e discentes do Curso de Enfermagem da UFPI, em uma maternidade pública de referência no atendimento à mulher no ciclo gravídico-puerperal no estado do Piauí. O cenário foi uma maternidade pública localizada na região sul da cidade de Teresina.

Este estudo apoiou-se em autores que trabalham com a temática, utilizando-se artigos científicos publicados, dissertações, livros e legislação do exercício profissional, como as resoluções do Conselho Federal de Enfermagem. Foram analisados dados referentes à implantação da SAE descritos por docentes e discentes de enfermagem da UFPI, assim como por profissionais de enfermagem da instituição, desde 2005 até a atualidade. Este trabalho foi realizado a partir de relatórios de projetos de extensão, além de pesquisas científicas decorrentes de trabalhos de iniciação cientifica, conclusão de curso, mestrado em enfermagem e pesquisas realizadas pelos próprios docentes do referido departamento.

\section{RESULTADOS E DISCUSSÃO}

Os resultados do estudo abordam os aspectos relacionados às dificuldades encontradas para a implantação da SAE em uma maternidade pública de Teresina.

Mesmo com o respaldo legal, com abordagem nos currículos dos Cursos de Enfermagem no estado do Piauí desde a década de 1980, e com a temática frequente em eventos científicos da área de Enfermagem, a prática da SAE ainda não foi implantada na sua totalidade nas instituições de saúde do Estado.

O currículo do Curso de Enfermagem da UFPI contempla a SAE em disciplina específica, e em todas as disciplinas profissionalizantes as docentes trabalham a SAE. Já foram realizados eventos destinados a docentes e discentes do Curso com a temática sobre $\mathrm{SAE}$, com a participação de especialistas convidados de outros estados, mas sua aplicação ainda é pequena e descontínua nas instituições onde ocorre a prática discente.

Nesse sentido, uma pesquisa desenvolvida em 2004 mostrou que os egressos da UFPI, em sua maioria, não desenvolvem a prática no cotidiano de suas atividades profissionais, mesmo que ao longo de 25 anos de curso tenham sido estudadas teorias de enfermagem e metodologia dos cuidados baseados nas necessidades humanas básicas ${ }^{(7)}$.

Essa realidade mostra a desarticulação entre ensino e prática profissional, com pouco impacto da academia para a transformação das instituições de saúde na prestação de cuidados. Tal prática é fragmentada e descontínua, comprometendo assim a qualidade do ensino e da assistência no estado do Piauí(8).

Buscando superar a desarticulação entre ensino e prática profissional, as docentes da disciplina Saúde da Mulher, da UFPI, desenvolvem desde 1985 o projeto de extensão "Assistência Humanizada à mulher no ciclo gravídico-puerperal", na maternidade de referência do Piauí, que também funciona como hospital escola na área de atenção à mulher no ciclo gravídico-puerperal. Esse projeto reconhece a necessidade de ações que garantam a implantação, de forma consistente, da SAE na Maternidade. Posteriormente foi elaborado outro projeto de extensão 
intitulado: "Implantação da SAE em uma maternidade pública em Teresina/PI", introduzido a partir de 2005 em uma ala de tratamento clínico da referida instituição, objetivando uma prática sistematizada e qualificada.

Este projeto teve a coordenação e subcoordenação de duas professoras da área da Saúde da Mulher do Departamento de Enfermagem da UFPI, além da participação de discentes do curso de Enfermagem da UFPI, enfermeiras, técnicas e auxiliares de enfermagem do setor.

Houve, a priori, capacitação em SAE para os profissionais e discentes de enfermagem, com a participação do Conselho Regional de Enfermagem do Piauí (COREN - PI) e apoio da chefia do departamento de enfermagem da UFPI, do gestor e da gerência de enfermagem da referida instituição. $E$ a seguir todo o planejamento inicial, incluindo treinamento dos recursos humanos; elaboração de normas e rotinas; elaboração dos impressos; organização dos recursos materiais e equipamentos e tudo o mais necessário para sua implantação.

Porém, é importante ressaltar que, ao ser iniciada a operacionalização do Projeto, as etapas do Processo de Enfermagem eram executadas apenas pelos docentes e discentes quando ocorriam as práticas e estágios da disciplina Saúde da Mulher. Aconteciam, portanto, de maneira fragmentada e descontínua.

Semestralmente eram realizados relatórios de acompanhamento e, para avaliar seus resultados, foram realizados vários estudos. $O$ estudo sobre a implementação da SAE na maternidade de referência, ao avaliar a utilização dos impressos da SAE em 673 prontuários, identificou que em $50,89 \%$ deles havia três impressos do Processo de Enfermagem implantado, sendo 68,7\% históricos de enfermagem completos, $86,6 \%$ evoluções e prescrições de enfermagem incompletas. Quanto à sua aplicação, obteve-se o seguinte resultado: $92,5 \%$ consideraram os históricos ótimos; as evoluções oscilaram entre boas $(43,5 \%)$ e ótimas $(31,2 \%)$; e as prescrições de enfermagem foram consideradas regulares por $54,7 \%$ dos pesquisados. As autoras concluíram que a SAE apresenta obstáculos e que deve haver investimentos no que se refere a treinamento e capacitação( ${ }^{(9)}$.

$\mathrm{Na}$ pesquisa "Percepção de auxiliares e técnicos de enfermagem sobre a SAE implantada em uma maternidade escola", as autoras chegaram às seguintes conclusões: a assistência apresenta-se deficiente, devido ao não envolvimento de toda a equipe, prejudicando a continuidade da assistência; falta de comunicação entre equipe e acadêmicos de enfermagem; falta de apoio da gerente de enfermagem e enfermeiras; e falta de educação permanente. Ressaltaram ainda que os profissionais desconheciam aspectos da SAE; alguns não participavam das etapas por falta de treinamento, mas reconheciam que deveriam estar inseridos no processo ${ }^{(10)}$.

Também em pesquisa realizada nessa mesma maternidade sobre a "Aplicabilidade da SAE em Unidade de Terapia Intensiva de um hospital escola", foram reveladas como dificuldades: o despreparo ou desconhecimento dos profissionais sobre o processo; falta de interesse e vontade das instituições; acomodação e falta de motivação dos profissionais; número insuficiente de pessoal; instabilidade e inconsciência dos pacientes; estresse e outros $^{(11)}$.

Os resultados desses estudos deixam evidente a trajetória percorrida pelos docentes, discentes e profissionais de enfermagem na implantação da SAE na instituição pesquisada, e apontam as dificuldades vivenciadas nessa caminhada. Assim, a partir da realidade conhecida, pode-se implementar o planejamento de estratégias para sua superação e a redefinição de rumos na implantação e implementação da SAE por discentes e profissionais que atuam em diferentes setores na maternidade.

Durante a realização dos projetos de extensão na maternidade de referência do Piauí foram apontadas como dificuldades: o não preenchimento dos impressos, a falta de envolvimento da equipe, a descontinuidade da assistência, a falta de comunicação entre equipe e acadêmicos de enfermagem, a falta de apoio de enfermeiras, a deficiência da educação permanente, o despreparo dos profissionais, o desinteresse das instituições, e o número insuficiente de pessoal.

Autores apontam como dificuldade encontrada na implantação da SAE a interdisciplinaridade, pois os outros profissionais de saúde não entendem que a enfermagem é uma profissão com autonomia e capacidade de cuidar baseada em princípios técnico-científicos ${ }^{(8)}$.

No que tange o ensino do processo de enfermagem existem outras dificuldades: dicotomia ensino/serviço, deficiência na articulação entre as fases que compõem o processo, e fragilidade dos conhecimentos relacionados às teorias de enfermagem e ao próprio processo de enfermagem ${ }^{(12)}$.

Considera-se também que a ausência de estímulo e apoio de instâncias superiores representa vulnerabilidade, pois está associada ao não envolvimento, não compromisso, não valorização de sua execução na prática, além da não capacitação para a execução da $\mathrm{SAE}$, bem como lacunas na formação pelo ensino formal e dificuldades estruturais institucionais ${ }^{(13)}$.

Então, torna-se necessário que os enfermeiros passem a desenvolver pesquisas e sejam sensíveis ao refletir e compreender a importância da aplicabilidade do processo de enfermagem no cotidiano de sua prática assistencial em todos os setores $^{(14)}$.

No que se refere às perspectivas de implantação/implementação 
da SAE em uma maternidade de referência em Teresina, os resultados desses estudos deixam evidente que essa realidade necessita ser revertida.Talé o objetivo de docentes da Universidade Federal do Piauí (UFPI), que realizam constantemente projetos de extensão e estudos científicos neste setor, incentivando a implantação da SAE por discentes e profissionais que atuam em diferentes setores na maternidade.

A partir dos resultados dos estudos foram planejadas atividades para superar as dificuldades, dentre as quais: apresentação do resultado das pesquisas realizadas para gestores, profissionais da instituição e profissionais de enfermagem em Seminário Institucional; realização de eventos específicos sobre SAE e sobre anotações de enfermagem, oficinas para capacitação e treinamento de profissionais e acadêmicos de enfermagem estagiários da instituição; reuniões com participação do COREN-PI para discutir as implicações éticas e legais do exercício profissional de enfermagem; e parceria com a Associação Brasileira de Obstetrizes e Enfermeiros Obstetras para a realização de eventos científicos sobre a atenção à mulher durante o ciclo gravídico- puerperal com enfoque na SAE.

\section{CONSIDERAÇÕES FINAIS}

No presente estudo, observaram-se dificuldades e resistência por parte da equipe de enfermagem para a implantação da SAE. No entanto, esses problemas podem ser solucionados a partir de um esforço conjunto por parte da equipe e do incentivo institucional.

Além disso, as instituições de ensino e os campos de prática devem estabelecer laços, proporcionando ao aluno um contexto prático para a formação de profissionais qualificados, já que a responsabilidade pela qualidade da formação dos (as) enfermeiros (as) não é somente das instituições formadoras, mas também dos órgãos prestadores da assistência à saúde.

Mediante o exposto, há necessidade de uma maior coesão da equipe de enfermagem como um todo, superando as dificuldades para a tomada de decisão, já que, para a aplicação do processo de enfermagem, todos os profissionais devem estar envolvidos e comprometidos, tendo em vista os benefícios para a profissão, cliente, profissional e instituição de saúde.

Espera-se, com este relato de experiência, trazer contribuições aos profissionais de enfermagem do Brasil como um todo, e em especial os do Piauí, no sentido de assegurar à clientela uma assistência digna, humanizada, sistematizada e de qualidade.

\section{Referências}

1. Possari JF. Prontuário do paciente e os registros de enfermagem. $2^{\mathrm{a}}$ ed.

São Paulo: Látria; 2008.

2. Amorim MMR, Katz L, Avila MB, Araújo DE, Valença M, Albuquerque CJM et al. Perfil das admissões em uma unidade de terapia intensiva obstétrica de uma maternidade brasileira. Rev Bras Saúde Mater Enfant. 2006;6(1):55-62. 3. Bittar DB, Pereira LV, Lemos RCA. Sistematização da assistência ao paciente crítico: proposta de instrumento de coleta de dados. Texto Contexto Enferm. 2006;15(4):617-28.

4. Conselho Federal de Enfermagem. Resolução COFEN n.272, de 27 de agosto de 2002. Sistematização da Assistência de Enfermagem (SAE) nas instituições de Saúde. Rio de Janeiro: COFEN; 2002.

5. Conselho Federal de Enfermagem. Resolução COFEN n 358, de 15 de Outubro de 2009 - Dispõe sobre a Sistematização da Assistência de Enfermagem e a implementação do Processo de Enfermagem em ambientes, públicos ou privados, em que ocorre o cuidado profissional de Enfermagem, e dá outras providências. Brasília: COFEN; 2009. 6. Martins SK, Kalinowski CE. Revendo o enfoque educativo no processo de aleitamento materno. In: Zagonel IPS, Lacerda MR, Lopes MGD, organizadoras. Experiência de enfermeiros da Secretaria Municipal de Saúde de Curitiba: subsídios para a sistematização do processo de cuidar em saúde coletiva. Associação Brasileira de Enfermagem - Curitiba: ABEn; 2004. 7. Avelino FPSD. O ensino da sistematização da assistência na visão do egresso da graduação em Enfermagem [dissertação]. Teresina (PI):
Universidade Federal do Piauí; 2004

8. Gonçalves LRR, Nery IS, Nogueira LT, Bonfin EG. O desafio de implantar a sistematização da assistência de enfermagem sob a ótica de discentes. Esc Anna Nery Rev Enferm. 2007;11(3):459-65.

9. Cardoso DV, Santiago MP, Viana LMM, Avelino FPSD. Sistematização da assistência de enfermagem em uma maternidade pública de Teresina-PI. In: Anais do VII congresso brasileiro de enfermagem obstétrica e neonatal; 2009 Jun 24-26; Teresina, BR. Teresina: ABENFO-PI; 2009.

10. Martins DS, Leite NAC. Percepção de auxiliares e técnicos de

enfermagem sobre a Sistematização da Assistência de Enfermagem implantada em uma maternidade escola [monografia]. Teresina (PI): Universidade Federal do Piauí; 2008.

11. Gonçalves FKO, Alvarenga RI, Avelino FPSD. Aplicabilidade da SAE para os enfermeiros de uma Unidade de Terapia Intensiva de um hospital escola [monografia]. Teresina (PI): Universidade Federal do Piauí; 2008. 12. Amorim FCM. O ensino do processo de enfermagem sob a ótica docente [dissertação]. Teresina (PI): Universidade Federal do Piauí; 2009. 13. Meneses SRT, Priel MR, Pereira LL. Autonomia e vulnerabilidade do enfermeiro na prática da sistematização da assistência de enfermagem. Ver Esc Enferm USP. 2011;45(4):953-8.

14. Freitas MC, Queiroz TA, Souza JAV. O processo de enfermagem sob a ótica das enfermeiras de uma maternidade. Rev Bras Enferm. 2007;6(2):207-12. 